\title{
The effects of removing dead bacteria by propidium monoazide on the profile of salivary microbiome
}

Qidi Ren ${ }^{1 \dagger}$, Fangqiao Wei ${ }^{1 \dagger}$, Chao Yuan ${ }^{1}$, Ce Zhu ${ }^{1,2}$, Qian Zhang ${ }^{3}$, Junkang Quan ${ }^{1}$, Xiangyu Sun ${ }^{*}$ and Shuguo Zheng ${ }^{1^{*}}$

\begin{abstract}
Background: Oral microbiome played an important role in maintaining healthy state and might exhibit certain changes under circumstances of diseases. However, current microbiological research using sequencing techniques did not regard dead bacteria as a separate part, causing findings based on subsequent analyses on dynamic equilibrium and functional pathways of microbes somewhat questionable. Since treatment by propidium monoazide (PMA) was able to remove dead bacteria effectively, it would be worth studying how the sequencing results after PMA treatment differed from those focusing on the whole microbiota.
\end{abstract}

Methods: Unstimulated whole saliva samples were obtained from 18 healthy people from 3 age groups (children, adults, and the elderly). After removal of dead bacteria by propidium monoazide (PMA), changes in the profile of salivary microbiome were detected using 165 rRNA sequencing technology, and differences among age groups were compared subsequently.

Results: Dead bacteria accounted for nearly a half of the whole bacteria flora in saliva, while freezing had little effect on the proportion of deaths. After treatment with PMA, the numbers of OTUs reduced by $4.4-14.2 \%$, while the Shannon diversity indices decreased significantly $(P<0.01)$. Only $35.2 \%$ of positive and $6.1 \%$ of negative correlations were found to be shared by the whole microbiota and that with dead bacteria removed. Differences in significantly changed OTUs and functional pathways among different age groups were also observed between the group of PMA and the control.

Conclusions: It was necessary to take the influence of living state of bacteria into account in analytic studies of salivary microbiome.

Keywords: Salivary microbiome, Propidium monoazide, Removal of dead bacteria, 16 S rRNA sequencing

*Correspondence: allon627@163.com; kqzsg86@bjmu.edu.cn

${ }^{\dagger}$ Qidi Ren and Fangqiao Wei contribute equally to this work and share the first authorship

${ }^{1}$ Department of Preventive Dentistry, Peking University School and Hospital of Stomatology \& National Center of Stomatology \& National Clinical Research Center for Oral Diseases \& National Engineering Laboratory for Digital and Material Technology of Stomatology, Beijing, People's Republic of China

Full list of author information is available at the end of the article

\section{Background}

Oral microbiome was highly complex, constituting the second most diverse microbiota throughout the human body [1]. These bacteria had closely associated with a series of oral and systemic diseases [2]. However, there were a lot of dead bacteria in the oral cavity, including those introduced by food intake and those existed in dental plaque. Normally, diseases could only be caused by living microbes that were able to replicate [3]. Live 
bacteria also play important biological processes such as digestion, absorption [4] and immune responses [5, 6]. This implied that the living state of bacteria might be an important factor of various physiological and pathological processes in the oral cavity.

In the past decades, great achievements had been made in research of human microbiome using DNA sequencing method, particularly for its role in disease conditions. Nevertheless, when we performed highthroughput sequencing analyses on oral samples, the dead microbes were not separated and would be also involved in the final results. This could cause inaccuracy in our understanding of the actual composition and functional pathways of microbial communities. In other words, DNA-based identification methods could not distinguish the DNA of living microbial cells (dormant cells, and growing or non-growing metabolically active cells) from dead ones. In consideration of the slow decay rate of DNA in dead bacterial cells, DNAbased detection methods tended to overestimate the richness and abundance of bacteria in samples. Therefore, the false-positive results of living pathogens made it doubtful for exploring relationship between the disease state and oral microbiota.

In this case, propidium monoazide (PMA) was introduced on account of its highly selective ability that could penetrate into the membranes of the damaged cells and covalently combined with the DNA in form of crosslinking [7]. PMA could effectively inhibit the amplification of DNA from the dead cells of both Gram-negative and Gram-positive bacteria [8-10] during the polymerase chain reaction (PCR) process prior to the following community sequencing procedures. PMA had been used in microbiological studies with regard to low-biomass cleanroom conditions [11], respiratory tract [12], and improvement of the counting accuracy of samples treated with sterilants $[13,14]$.

Saliva, as a pool of microorganisms with advantages of non-invasive and simple sampling procedures, had become a hotspot and representative of oral microbiome research $[15,16]$. One recent study [17] reported that PMA treatment had "subtle" effects on taxonomic composition of unstimulated saliva, which would be much less significant than the influences of temporal dynamics or individual specificity. Hence, the present study was designed to use $16 S$ rRNA sequencing technique to make comparisons between the whole bacteria flora in saliva and that with dead bacteria removed with PMA employed as the separating agent. The aspects of comparisons included not only the composition of salivary microbiome but also the correlations and functional pathways, as well as potential age-effects. In one word, the main purpose of our study was to investigate if removal of dead bacteria by PMA treatment had effects on the profile of salivary microbiome.

\section{Methods \\ Sample information}

Unstimulated whole saliva samples were collected from participants aged $4-6,18-30$, and $50-60$ years, with the exclusion criteria as follows: (1) who had taken antibiotics or probiotics within the last 4 weeks before sampling; (2) who were undergoing orthodontic treatment; (3) who could not cooperate with the study process. This study was approved by the ethics committee of Peking University School of Stomatology (PKUSSIRB-202056084). All participants had signed informed consent by themselves or their guardians before sampling procedures started.

\section{Exploration of the influence of freezing by staining of dead bacteria}

We first designed a pilot study to see if freezing had impact on the ratio of dead microbes. The sampling procedures began in the morning at 8:00-10:00 a.m., and no food, water, chewing gum or oral cleaning measures were taken within $2 \mathrm{~h}$ before sampling. After rinsing mouth with pure water thoroughly and having a rest for $10 \mathrm{~min}$, $2 \mathrm{ml}$ unstimulated whole saliva was collected from each of the 3 healthy participants aged $18-30$ years. These saliva samples were collected by 2 separate tubes $(1 \mathrm{ml}$ saliva in each tube) and labelled with different group names (Group Im and Group Fr, for grouping details please see below). All the samples were centrifuged at $5000 \mathrm{rpm} \times 10 \mathrm{~min}$, then the supernatant was discarded. Samples in Group Im were immediately stained with the LIVE/DEAD ${ }^{\mathrm{TM}}$ BacLight $^{\mathrm{TM}}$ Bacterial viability and counting Kit (Thermo Fisher Scientific, USA), whereas samples in Group Fr were kept in the refrigerator at $-80{ }^{\circ} \mathrm{C}$ for 5 days before the staining procedures began. According to the instructions, $2 \mathrm{ml}$ Component A (SYTO 9 dye) and $2 \mathrm{ml}$ Component B (Propidium iodide) were mixed in a microfuge tube thoroughly first, then the dye mixture was added to $\mathrm{ddH}_{2} \mathrm{O}$ with the concentration of $3 \mu \mathrm{l} / \mathrm{ml}$. Afterwards, $300 \mu \mathrm{l}$ of the prepared mixture was added to resuspend the precipitate. The solution was mixed thoroughly and incubate at room temperature in a dark environment for $15 \mathrm{~min} .20 \mu \mathrm{l}$ stained solution was placed on the slide, fixed by the cover slide, and observed under the fluorescence confocal microscope (Leica, TCS SP8, Germany; $\times 63$ with an additional $\times 5$ zoom) which excitation/emission wavelengths were set at 488/500-550 nm for SYTO 9 and 561/575-650 nm for propidium iodide. CLSM images were acquired by the software LAS $\mathrm{X}$ at a resolution of $512 \times 512$ pixels. Six $5 \times$ zoom fields of view were randomly selected, and the dead bacteria ratios were calculated by Image J 1.53 and compared between 
Group Im and Group Fr to see if freezing did affect the proportions of deaths.

\section{Sampling and preparation of saliva}

18 participants covering age groups of 4-6, 18-30, 50-60 years old were recruited ( 3 males and 3 females in each age group; Group C1 and P1: 4-6-year-olds as a representative of children; Group C2 and P2: 18-30-yearolds on behalf of adults; Group C3 and P3: 50-60-yearolds representing the elderly; see Additional file 1: Table S1 for more information). "Group C" represented the mixture of Group C1, C2 and C3, while "Group P" was the summary of Group P1, P2 and P3.

Unstimulated whole saliva was selected as the sample for the present study to avoid any potential effects brought by extraneous stimulating method. The sampling procedures also began in the morning at 8:00-10:00 a.m., and no food, water, chewing gum or oral cleaning measures were taken within $2 \mathrm{~h}$ before sampling. After rinsing mouth with pure water thoroughly and having a rest for $10 \mathrm{~min}, 2 \mathrm{ml}$ unstimulated whole saliva was collected from each participant. These saliva samples were collected by 2 separate tubes ( $1 \mathrm{ml}$ saliva in each tube), labelled with different group names (Group $\mathrm{C}$ for control, and Group P for PMA treatment) and placed on ice at once.

\section{PMA treatment and DNA extraction}

In Group P, saliva samples were centrifuged at $7000 \mathrm{rpm}$ for $5 \mathrm{~min}$, then the supernatant was discarded and the precipitate were rinsed with $1 \mathrm{ml}$ PBS for once. A small amount of the sample was extracted and counted under microscope, then the sample was diluted with $1 \times$ PBS to ensure that the concentration of bacteria was around $1 \times 10^{7}-2 \times 10^{7} / \mathrm{ml} .1 .25 \mu \mathrm{l}$ PMA solution $(20 \mathrm{mM})$ was added to $500 \mu$ l diluted saliva sample, then the mixture was incubated for 5 min without light under intermittent oscillation and placed under a $650 \mathrm{~W}$ halogen lamp for 3 min with a distance of $6 \mathrm{~cm}$ away from the light source. The PMA-treated samples were centrifuged at $7000 \mathrm{rpm}$ for $5 \mathrm{~min}$ and the supernatant was removed again, and the precipitate was rinsed with $1 \mathrm{ml}$ PBS for once before genomic DNA extraction in terms of the research protocol by Sweet et al. [18].

\section{PCR amplification and sequencing}

The sequence of the V3-V4 region of $16 S$ rRNA was analysed by high-throughput sequencing. A two-step PCR amplification method was used to construct the library. The purified DNA was used as a template to be amplified by $16 S$ rRNA V3-V4 region universal primer 357F (5'-ACTCCTACGGRAGGCAGCAG-3') and 806R (5'GGACTACHVGGGTWTCTAAT- ${ }^{\prime}$ ) and detected via
$1.2 \%$ agarose gel electrophoresis. The samples with good detection efficiency were recycled from the agarose gel electrophoresis, and 8 cycles of PCR amplification were carried out with the recovered product as a template. The sequences needed for Illumina platform sequencing was added to both ends of the target fragment. All the PCR products were recycled by AxyPrepDNA gel Recovery Kit (AXYGEN company) and quantified by FTC-3000TM Real-Time PCR. After the mole ratio was mixed, the library was constructed and sequenced on the Illumina platform.

The initial PCR mixtures contained $5 \times$ Buffer $10 \mu \mathrm{l}$, dNTP $(10 \mathrm{mM}) 1 \mu \mathrm{l}$, Phusion super fidelity DNA polymerase $1 \mathrm{U}$, forward and reverse primers $(10 \mathrm{mM}) 1 \mu \mathrm{l}$, template DNA 20-50 ng, supplemental ultra-pure water to $50 \mu \mathrm{l}$. Thermal cycling consisted of an initial denaturation step at $94{ }^{\circ} \mathrm{C}$ for $2 \mathrm{~min}$, followed by 24 cycles of denaturation $94{ }^{\circ} \mathrm{C}$ for $30 \mathrm{~s}$, annealing at $56{ }^{\circ} \mathrm{C}$ for $30 \mathrm{~s}$, and extension at $72{ }^{\circ} \mathrm{C}$ for $30 \mathrm{~s}$, with a final extension step at $72{ }^{\circ} \mathrm{C}$ for $5 \mathrm{~min}$. The secondary PCR mixtures contained $5 \times$ Buffer $8 \mu$ l, dNTP $(10 \mathrm{mM}) 1 \mu \mathrm{l}$, Phusion super fidelity DNA polymerase $0.8 \mathrm{U}$, positive and reverse primers $(10 \mathrm{mM}) 1 \mu \mathrm{l}$, template DNA $5 \mu \mathrm{l}$, supplement ultra-pure water to $40 \mu \mathrm{l}$. Thermal cycling had similar procedures with the above, but the number of cycles were 8 and a heat preservation step at $10{ }^{\circ} \mathrm{C}$ was supplemented in the end.

\section{Analysis of operational taxonomic units (OTUs) profile}

The raw sequences were processed to concatenate reads into tags according to their overlaps, after which reads belonging to each sample were separated with barcodes and low-quality reads were removed. The processed tags were clustered, and chimaeras were removed prior to analysis. Operational taxonomic units (OTUs) were defined at $97 \%$ sequence similarity to taxa by matching to the Silva128 database using mothur (classify.seqs) software with a confidence threshold of 0.6.

\section{Statistical analysis}

The Venn diagrams were drawn by jvenn method [19]. For alpha diversity analysis, nonparametric tests (Wilcox rank-sum test for two groups and Kruskal-Wallis test for multiple groups) were used, and the results were drawn by the boxplot package of $\mathrm{R}$ software (version: 3.6.1). For beta diversity analysis, UniFrac method was used to obtain the distance matrix between samples, and the multi-sample similarity tree was drawn using the dendextend package of R. Also, PERMANOVA (vegan-version: 2.5-5, function: adnois) was conducted in $\mathrm{R}$ software with gender and age group selected as covariates to compare the difference. 
At the genus level, the ggplot2 package of $\mathrm{R}$ was employed to plot the column chart and draw the column diagram of microbiota. Genus count table was used as an input for SparCC. The SparCC analysis was conducted with composition-robust correlations from the median of 20 iterations, while 1000 bootstrap samples were used to infer pseudo $P$ values with SpiecEasi (version 1.1.1) of $\mathrm{R}$. The inferred correlations were restricted to those with a correlation co-efficient of more than 0.6 or less than $-0.6(P<0.05$, two-sided). Visualization of results was performed with igraph (version 1.2.5) package of R. The Picrust2 (phylogenetic investigation of communities by reconstruction of unobserved states) was used to identify differentially abundant OTUs and functional pathways.
The aldex2 (anova-like differential expression 2, version 1.18.0) package of $R$ was carried out to identify differentially abundant OUTs and functional pathways, with nonparametric tests used in comparisons between different groups. OTUs and functional pathways with a $P$ value of less than 0.05 were screened, and GGplot2 (version 3.3.2) package of $\mathrm{R}$ was used for visualization of the results.

\section{Results}

Influence of freezing on the dead ratio of salivary microbiota

Figure 1A showed representative fluorescence images of the living state of salivary microbiome, with a dead proportion of $32.8-71.7 \%$ (Fig. 1B). No significant difference

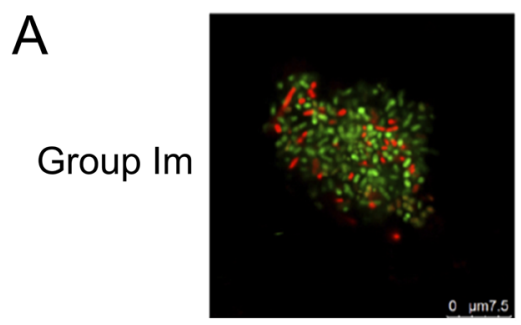

Participant 1

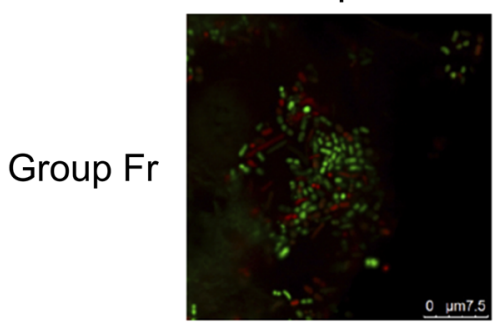

B

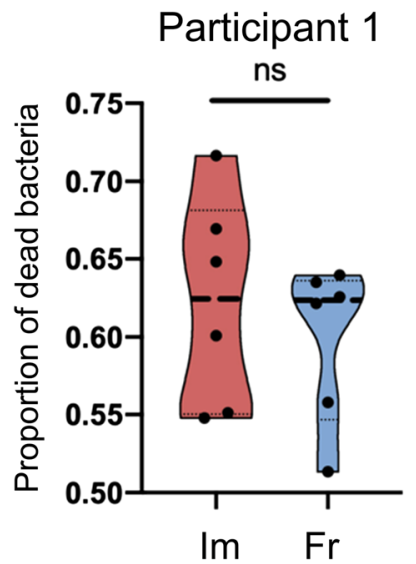

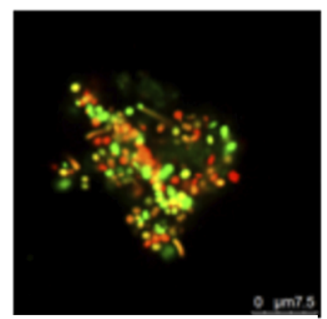

Participant 2

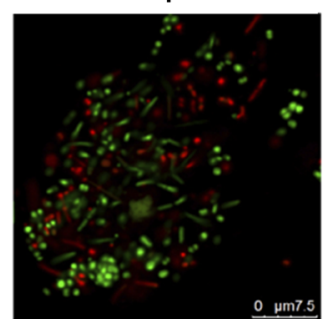

Participant 2

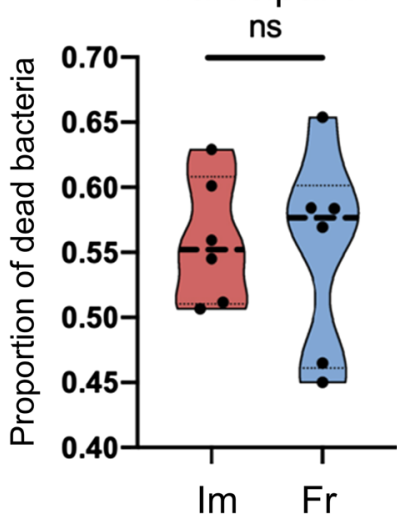

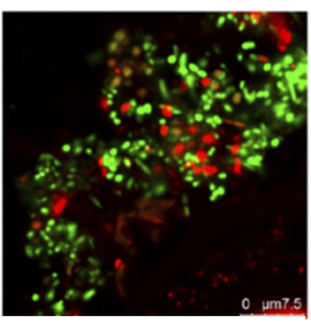
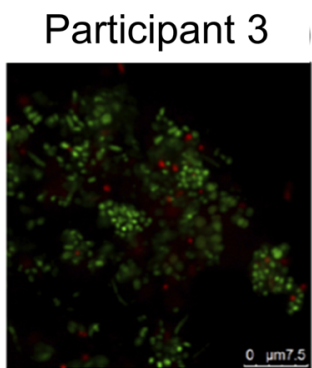

Participant 3

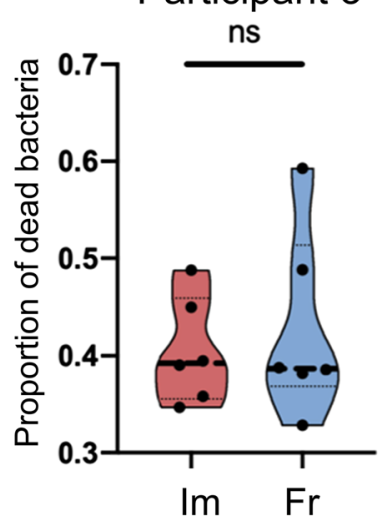

Fig. 1 The living state and dead proportion of salivary microbiome. A Fluorescence microscopic observation with staining immediately (upper row, Group Im) or after keeping in freezing condition $\left(-80^{\circ} \mathrm{C}\right.$ ) for 5 days (lower row, Group Fr). B The proportion of dead bacteria in the three participants ( $P$ values: $0.51,0.84,0.62)$. Error bars represented median \pm quartile $(n=6)$ 
was found between immediately stained samples and those undergone freezing condition.

\section{Changes in genera profile after PMA treatment}

Total number of raw reads per sample was provided in Additional file 1: Table S2, and the mean reads per sample after QC was 32301. The sequencing depth was analysed and a sparse curve was drawn (Additional file 1: Figure S1). After treated with PMA, the numbers of OTUs reduced by $4.4-14.2 \%$ (Fig. 2A and Additional file 1: Table S3). The Shannon index for $\alpha$-diversity decreased significantly $(P=0.006$, Fig. $2 \mathrm{~B})$ in all the three

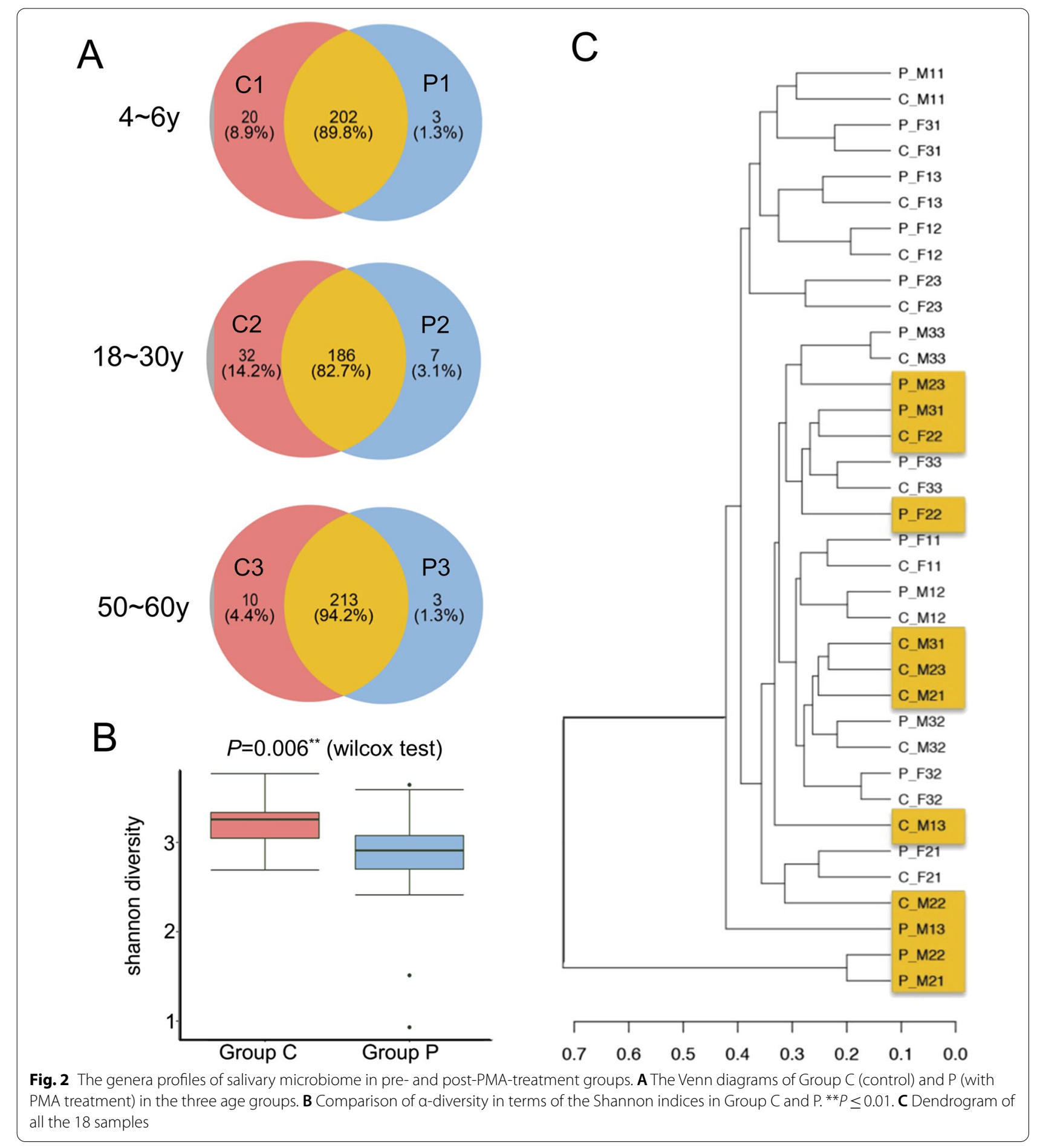


age groups. Two OTUs (Treponema sp.HMT_927 and Clostridiales_[F-1][G-1]bacterium_HMT_093) completely disappeared in Group P.

Comparative analysis based on the composition of genera in samples with and without PMA treatment showed that some samples derived from the same participant were separated with a distance in the cluster tree, indicating that the removal of dead bacteria had influence on the profile of salivary microbiome to a certain extent (Fig. 2C). The proportion of some high-abundance genera (e.g. Streptococcus, Neisseria, Veroniella, Rosella, Haemophilus, and Actinomycetes) increased at the genus level while that of some other relatively low-abundance bacteria decreased (Fig. 3).

The unweighted UniFrac-based PERMANOVA results showed that beta diversity was not significantly correlated with gender $(P=0.112)$, age subgroup $(P=0.589)$ and PMA treatment $(P=0.435)$, but significantly correlated with age $(P=0.002)$ among individuals (Additional file 1: Table S4).

\section{Different patterns in co-occurrence networks with PMA treatment}

Co-occurrence network analysis was carried out to investigate the variations in relationships between different genera after using PMA (Fig. 4), which results revealed that there were dramatic changes in their correlations. Only 63 pairs in 179 positive correlations and 2 in 33 negative correlations were shared by preand post-PMA-treatment groups (i.e., Group $C$ and P) under the circumstance of absolute value of correlation coefficient $\geq 0.6$ and $P$ value $<0.05$. A larger number of positive correlations among some genera (Butyrivibrio, Saccharibacteria_(TM7)_[G-3], Leptotrichia, Peptostreptococcus, Veillonella, Selenomonas, Atopobium, Peptostreptococcaceae_[XI][G-1], Lachnoanaerobaculum, Actinomyces, and Mogibacterium) and fewer negative correlations (mainly related to Treponema and Capnocytophaga) were found in Group P compared with Group C.

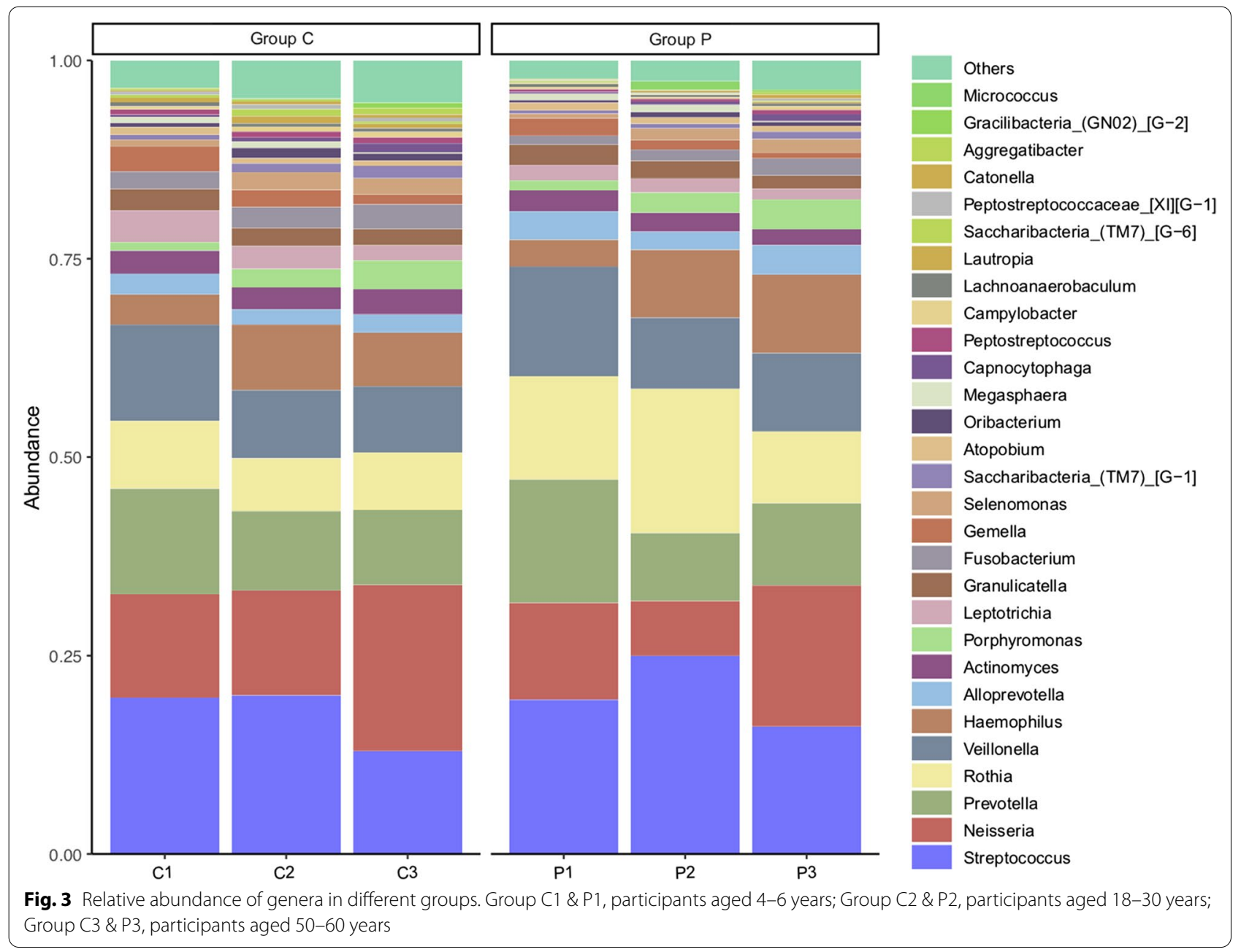




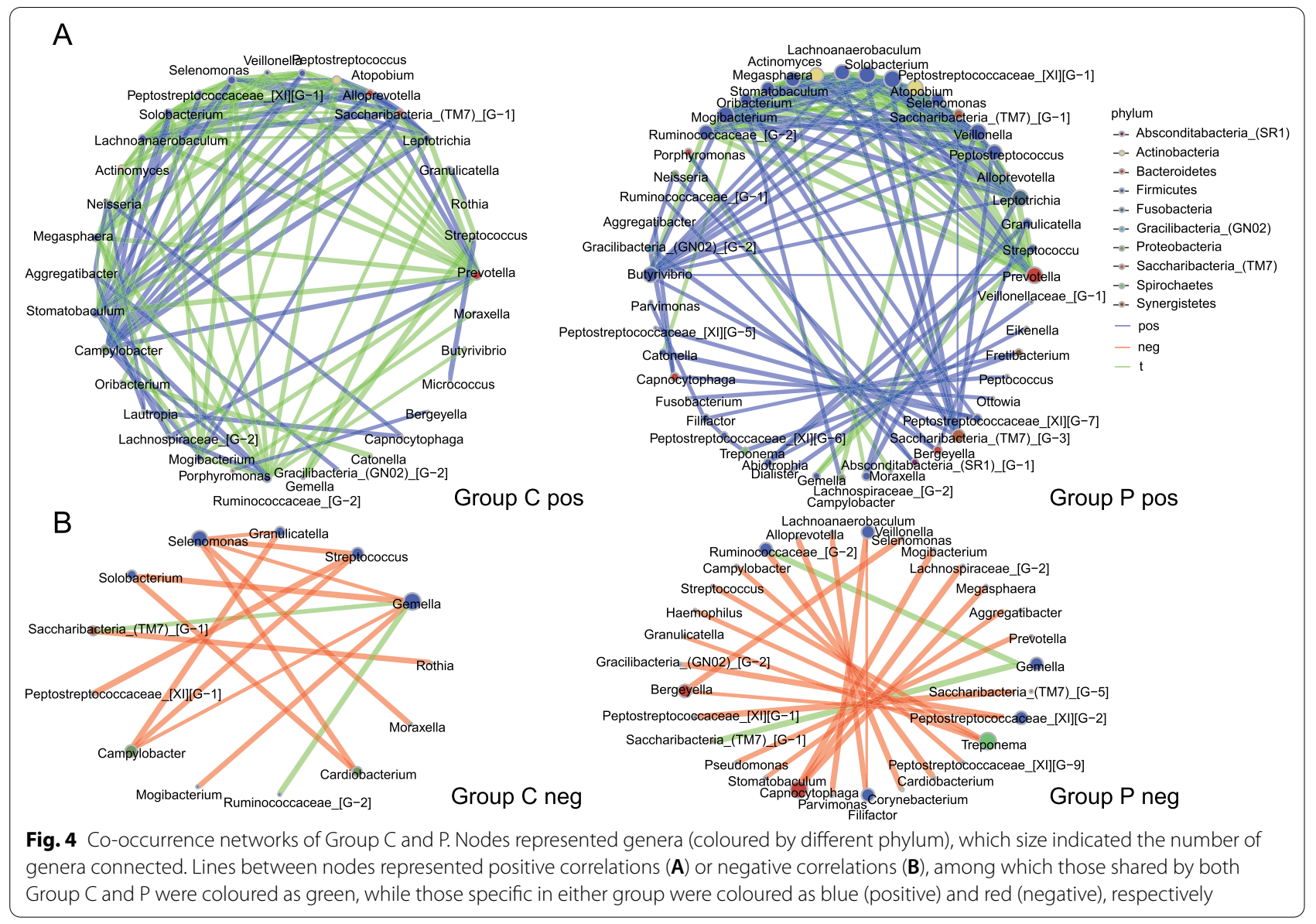

\section{Differences among the three age groups}

Potential age-effects were explored by comparison among the three age groups in Group P and C. At the OTU level, some OTUs had significant differences in relative abundance between Group $\mathrm{C} 1$ and C2 (Stomatobaculum sp.HMT 097, Lachnospiraceae [G-3] bacterium HMT 100), Group C1 and C3 (Peptostreptococcaceae_[XI][G-5] bacterium HMT 493, Absconditabacteria_(SR1)_[G-1] bacterium HMT 345, Bergeyella sp. HMT 907, Comamonadaceae sp. HMT 894, Treponema sp. HMT 238, Leptotrichia sp. HMT 218, Haemophilus sp. HMT 036, Streptococcus salivarius, Campylobacter rectus, Capnocytophaga granulosa, Lachnospiraceae_[G-2] bacterium HMT 096, Porphyromonas endodontalis, Granulicatella elegans, Prevotalla aurantiaca), and Group C2 and C3 (Ottowia sp. HMT 894, Selenomonas noxia). After PMA treatment, OTUs which had significant differences in relative abundance became partly different in comparisons between Group P1 and P2 (Stomatobaculum sp.HMT 097), Group P1 and P3 (Ottowia sp. HMT 894, Leptotrichia sp. HMT 218, Actinomyces sp. HMT
169, Streptococcus salivarius, Campylobacter rectus, Prevotella intermedia, Gracilibacteria_(GN02) [G-2] bacterium HMT 873, Porphyromonas endodontalis, Prevotella aurantiaca), and Group P2 and P3 (Actinomyces sp. HMT 180, Alloprevotella rava, Prevotella oris) (Fig. 5).

As for functional pathways, no significant difference was present in the comparison between Group $\mathrm{C} 1$ and $\mathrm{C} 2$, as well as that between Group C2 and C3, while three pathways (Phosphotransferase system-ko02060, Benzoate degradation-ko00362, Ascorbate and aldarate metabolism-ko00053) exhibited significant differences between Group C1 and C3. After PMA treatment, only one pathway was found to have significant differences in the comparison between Group P1 and P2 (Biofilm formation Escherichia coli-ko02026), so as that between Group P2 and P3 (Phosphotransferase system-ko02060). Six pathways (Starch and sucrose metabolism-ko00500, Lysine biosynthesis-ko00300, Glycolysis / Gluconeogenesis-ko00010, Fructose and mannose metabolism-ko00051, Cysteine and methionine metabolism-ko00270, Ascorbate and aldarate 


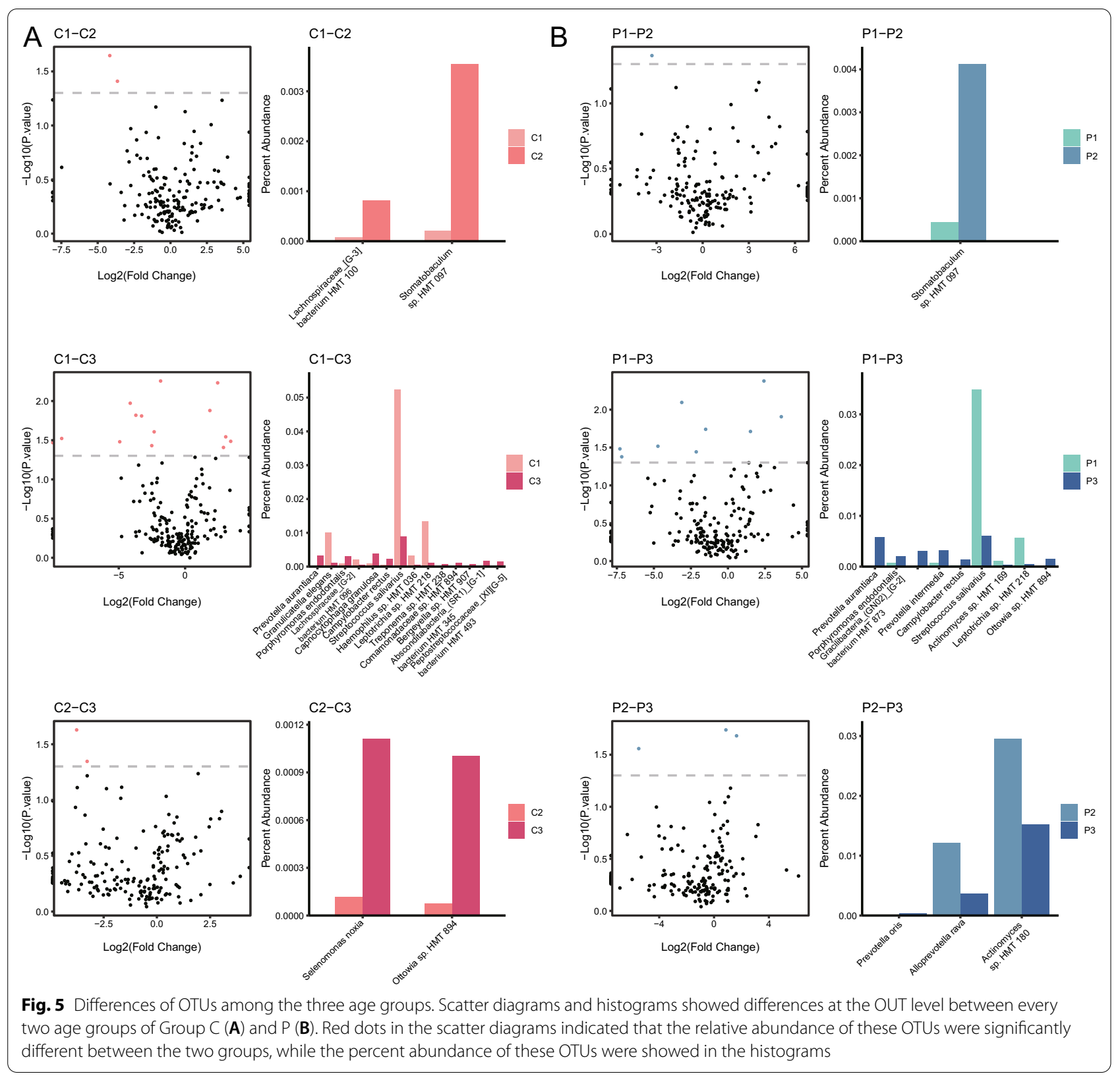

metabolism-ko00053) had significant differences in the comparison between Group P1 and P3 (Fig. 6).

\section{Discussion}

The $16 S$ rRNA approach was a popular technique for microbiome research, however less attention was paid to the living state of bacteria which might potentially affect the results. PMA was able to remove dead microbes by covalently binding with DNA, while the viable cells, including viable-but-non-culturable (VBNC) cells which had intact cell membranes, would not be significantly affected under certain PMA concentration ranges [20]. As PMA was already used in some in-vitro studies and exhibited acceptable antibacteria effectiveness [21, 22], while one recent study reported that PMA treatment had "subtle" effects on taxonomic composition of unstimulated saliva and would be much less significant than the influences of temporal dynamics or individual specificity [17], we designed the present study to justify if removal of dead bacteria with PMA treatment could give rise to changes not only in the composition of salivary microbiome but also in the correlations and functional pathways, as well as presence of potential age-effects. Since the saliva 

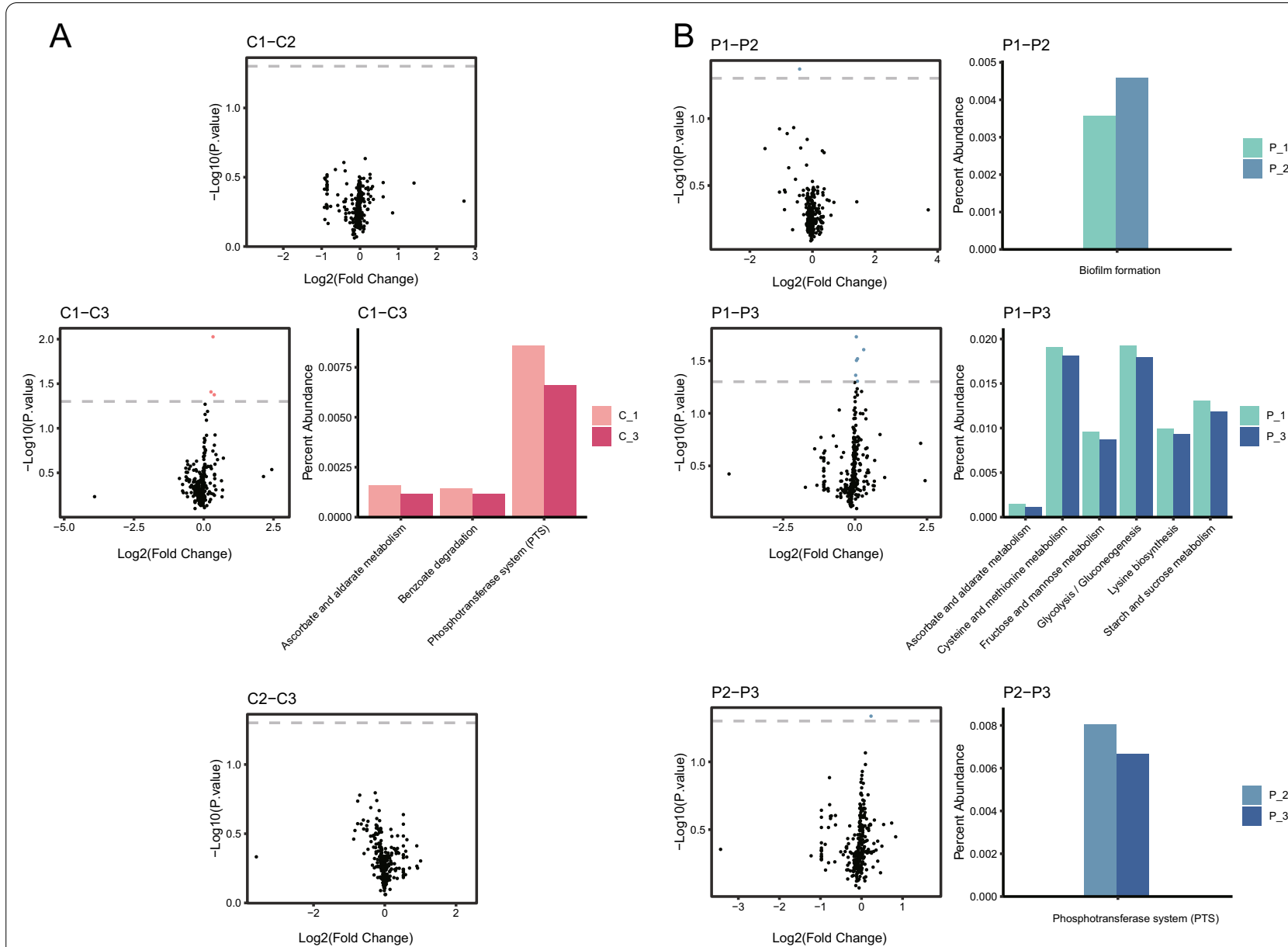

Fig. 6 Differences of functional pathways among the three age groups. Scatter diagrams and histograms showed differences at the pathway level between every two age groups of Group C (A) and P (B). Red dots in the scatter diagrams, same as Fig. 5. The percent abundance of these pathways were also showed in the histograms

samples might have a frozen-thawed procedure on various occasions prior to further examinations, we compared the dead ratios of salivary microbiota between fresh saliva sample and that with freezing storage via a pilot study with staining methods, which verified that freezing condition contributed little to the proportion of dead bacteria.

To our knowledge, many bacteria could not be cultured in vitro, resulting in a failure of simulating the actual status of living microbiota by cultivation only [23]. In the meantime, the rRNA analysis, though was used as a general indicator of active microorganisms in environmental samples, could be ineffective in certain cases [24]. It was confirmed by several previous studies that PMA was effective in sterilization of samples, during which process dead bacteria were almost completely removed with only less than $0.27 \%$ left [25-27] and without evident loss and decomposition of living bacteria [28], making PMA treatment reasonable and acceptable for separating bacteria based on the living state to keep living bacteria only in the following analysing procedures.

Besides PMA, Ethidium monoazide (EMA) [29], Surface-Enhanced Raman Scattering (SERS) [30], hand-held double monochromator [31] and RNA-based $16 S$ rRNA sequencing [32] could also be used to separate or remove dead bacteria. Among them, high concentration of EMA might enter the whole cells of some species, causing the lack of specificity for intact bacterial cells [7]. SERS could quantify the percentage of dead bacteria, but could not distinguish different genera [30]. Also, hand-held double monochromator had limited capacity in distinguishing different genera in mixed oral samples. Although the detection of RNA (especially the highly unstable mRNA) tended to indicate the presence of living cells far better than the detection of DNA, the degradation of RNA and the complicated physiological status of the cells both made it challenging to be used in oral samples like saliva. In addition, false-positive signals from residual 
transcripts could occur in case of high level of dead bacteria [7]. Among all these methods, PMA treatment was easier to operate and had reliable effects in removal of dead bacteria, which should be appropriate to be chosen as the key agent for the present study.

Our results of changes in genera profile revealed that there were a considerable proportion of dead bacteria in the whole microbiota. However, different trends were observed in the changes of genera after PMA treatment. The proportion of some high-abundance genera increased, while that of relatively low-abundance bacteria decreased. These findings implied that the role of lowabundance genera might be exaggerated in sequencing analysis without removal of dead bacteria. Besides, we observed dramatic changes in co-occurrence networks between pre- and post-PMA-treatment groups, with a greater number of positive correlations among certain genera detected in the group of PMA. Selenomonas was found to be significantly associated with recurrent aphthous stomatitis (RAS) [33], gestational diabetes mellitus [34], and the presence of dental caries [35] and periodontitis [36]. Butyrivibrio exhibited significantly higher prevalence in fecal samples collected from patients with Parkinson's Disease [37]. Peptostreptococcus was significantly enriched in pathological samples from patients with oral squamous cell carcinoma or periodontitis [38]. For analyses of salivary microbiome without removing dead microbes, the functions of these genera above were much likely to be underestimated. Capnocytophaga, which was formerly considered as a part of normal flora in the oral cavity, still had broad negative correlations after PMA treatment, demonstrating its functional potentiality in maintaining the stability of microbiota in saliva.

Among the three age groups, differences in significantly changed OTUs and functional pathways in response to PMA treatment were also observed. For these OTUs, previous studies showed that Selenomonas noxia in saliva was associated with refractory periodontitis [39] and hepatitis C [40], while Capnocytophaga granulosa was associated with Papillon-Lefèvre syndrome [41], but the associations of Peptostreptococcaceae_[XI][G-5]_bacterium_HMT_493, Ottowia sp._HMT_894, Treponema sp._HMT_238, Lachnospiraceae_[G-2] bacterium HMT 096 and some other bacteria with diseases were still not clear. Some of these microbes might always come up in a dead form in saliva samples, resulting in a potential bias in analysis of the pathogenic process in the circumstances of diseases. Similar with a previous study which found certain pathways could change with age [42], we also found that some functional pathways exhibited an age difference. For example, the phosphotransferase system, which was a major mechanism used by bacteria for uptake of carbohydrates (particularly hexoses, hexitols, and disaccharides) [43], had significant differences between children (Group C1) and older people (Group C3) only before PMA treatment. Future studies with a larger number of samples would be beneficial to conduct more in-depth investigations on these age-varied pathways.

Although we confirmed the influence of removing dead bacteria by PMA on the profile of salivary microbiome in the present study, certain limitations should be kept in mind to carry forward future studies. First, saliva was selected as a representative habitat of oral microbiome in the present study, but other sites, such as dental plaque which also contained a large amount of dead bacteria [44], yet need further exploration. Second, whether removal of dead bacteria had influence on the profile of microbiome in other sequencing methods (e.g. metagenomic analysis) still remained unrevealed. Also, further studies with RNA-based 16S amplicon library included would be a much important route to validate the effects of propidium monoazide treatment. Third, since our study was based on healthy individuals, the change of salivary microbiota and the impact of PMA under circumstances of diseases (including oral and systemic diseases) could also become a future direction of research in this field. Fourth, the sample size should be enlarged with qPCR quantification applied in future studies, so as to understand the specific reduction of each strain and to learn more about whether salivary microbiota of certain population group (e.g. 18-30-year-old men, who might have much lower sequence readouts as shown in Additional file 1: Figure S1) would have some particular characteristics and hence exhibit greater changes after PMA treatment.

\section{Conclusions}

In summary, differences in genus, diversity, correlations and functional pathways and distinctions among different age groups were found between the whole salivary microbiota and that with dead bacteria removed by PMA treatment, highlighting the potential significance of removing dead bacteria in high-throughput sequencing research. These findings enlightened us that it was necessary to take the influence of living state of bacteria into account in analytic studies of salivary microbiome.

\section{Abbreviations}

EMA: Ethidium monoazide; OTUs: Operational taxonomic units; PCR: Polymerase chain reaction; PMA: Propidium monoazide; RAS: Recurrent aphthous stomatitis; SERS: Surface-Enhanced Raman Scattering. 


\section{Supplementary Information}

The online version contains supplementary material available at https://doi. org/10.1186/s12903-021-01832-5.

Additional file 1. Table S1. Information of participants and samples. Table S2. Total number of reads per sample. Table S3. Comparison among the three age groups by OTUs present in Group $\mathrm{C}$ and absent in Group P. Table S4. Beta diversity analysis based on PERMANOVA. Figure S1. Rarefaction curves for each experimental sample.

\section{Acknowledgements}

We sincerely appreciate the saliva donors for their contributions to the present study.

\section{Authors' contributions}

QR, FW, XS, SZ contributed to conception, design, data acquisition, analysis, and interpretation, drafted and critically revised the manuscript; CY, CZ, QZ, JQ contributed to conception, design, and data interpretation, drafted and critically revised the manuscript. All authors approved to be accountable for all aspects of the work. All authors read and approved the final manuscript.

\section{Funding}

This work was supported by the National Natural Science Foundation of China (Grant number: 81801037), Beijing Municipal Science and Technology Commission (Grant number: Z181100001618015), and the Ministry of Science and Technology of the People's Republic of China (Grant number: 2018FY101005). These funding covered the expenditure on the sequencing of saliva samples and all the subsequent analyses. The three funding bodies were not involved in the design of the study and collection, interpretation of data and in writing the manuscript.

\section{Availability of data and materials}

The sequencing data from this study have been submitted to Sequence Read Archive (http://www.ncbi.n/m.nih.gov/sra/) under accession numbers PRJNA639033.

\section{Declarations}

\section{Ethics approval and consent to participate}

All procedures followed were in accordance with the Peking University School and Hospital of Stomatology Ethics Committee (PKUSSIRB-202056084) and with the Declaration of Helsinki. All participants had signed informed consent by themselves or their guardians before sampling procedures started.

\section{Consent for publication}

Not applicable.

\section{Competing interests}

The authors declare that they have no competing interests.

\begin{abstract}
Author details
1 Department of Preventive Dentistry, Peking University School and Hospital of Stomatology \& National Center of Stomatology \& National Clinical Research Center for Oral Diseases \& National Engineering Laboratory for Digital and Material Technology of Stomatology, Beijing, People's Republic of China. ${ }^{2}$ Department of Preventive Dentistry, Shanghai Jiao Tong University School of Dentistry, Shanghai Ninth People's Hospital, Shanghai, People's Republic of China. ${ }^{3}$ Central Laboratory, Peking University School and Hospital of Stomatology \& National Center of Stomatology \& National Clinical Research Center for Oral Diseases \& National Engineering Laboratory for Digital and Material Technology of Stomatology, Beijing, People's Republic of China.
\end{abstract}

Received: 9 March 2021 Accepted: 16 September 2021 Published online: 22 September 2021

\section{References}

1. Consortium HMP. Structure, function and diversity of the healthy human microbiome. Nature. 2012;486(7402):207-14.

2. Gao L, Xu T, Huang G, Jiang S, Gu Y, Chen F. Oral microbiomes: more and more importance in oral cavity and whole body. Protein Cell. 2018;9(5):488-500.

3. Walter A, Marz A, Schumacher W, Rosch P, Popp J. Towards a fast, high specific and reliable discrimination of bacteria on strain level by means of SERS in a microfluidic device. Lab Chip. 2011;11(6):1013-21.

4. Costalonga $M$, Herzberg MC. The oral microbiome and the immunobiology of periodontal disease and caries. Immunol Lett. 2014;162(2 Pt A):22-38.

5. Yu JC, Khodadadi H, Baban B. Innate immunity and oral microbiome: a personalized, predictive, and preventive approach to the management of oral diseases. EPMA J. 2019;10(1):43-50.

6. du Teil EM, Gabarrini G, Harmsen HJM, Westra J, van Winkelhoff AJ, van Dijl JM. Talk to your gut: the oral-gut microbiome axis and its immunomodulatory role in the etiology of rheumatoid arthritis. FEMS Microbiol Rev. 2019;43(1):1-18.

7. Fittipaldi M, Nocker A, Codony F. Progress in understanding preferential detection of live cells using viability dyes in combination with DNA amplification. J Microbiol Methods. 2012;91(2):276-89.

8. Nocker A, Cheung CY, Camper AK. Comparison of propidium monoazide with ethidium monoazide for differentiation of live vs. dead bacteria by selective removal of DNA from dead cells. J Microbiol Methods. 2006;67(2):310-20.

9. Bae S, Wuertz S. Survival of host-associated bacteroidales cells and their relationship with Enterococcus spp., Campylobacter jejuni, Salmonella enterica serovar Typhimurium, and adenovirus in freshwater microcosms as measured by propidium monoazide-quantitative PCR. Appl Environ Microbiol. 2012;78(4):922-32.

10. Nocker A, Camper AK. Novel approaches toward preferential detection of viable cells using nucleic acid amplification techniques. FEMS Microbiol Lett. 2009;291(2):137-42.

11. Vaishampayan P, Probst AJ, La Duc MT, Bargoma E, Benardini JN, Andersen GL, Venkateswaran K. New perspectives on viable microbial communities in low-biomass cleanroom environments. ISME J. 2013;7(2):312-24.

12. Cuthbertson L, Rogers GB, Walker AW, Oliver A, Green LE, Daniels TW, Carroll MP, Parkhill J, Bruce KD, van der Gast CJ. Respiratory microbiota resistance and resilience to pulmonary exacerbation and subsequent antimicrobial intervention. ISME J. 2016;10(5):1081-91.

13. Takahashi H, Kasuga R, Miya S, Miyamura N, Kuda T, Kimura B. Efficacy of propidium monoazide on quantitative real-time PCR-based enumeration of Staphylococcus aureus live cells treated with various sanitizers. J Food Prot. 2018;81(11):1815-20.

14. Xing-Long X, Cong-Cong L, Yang Q, Yi-Gang Y, Hui W. Molecular monitoring of Escherichia coli O157: H7 sterilization rate using qPCR and propidium monoazide treatment. Lett Appl Microbiol. 2013;56(5):333-9.

15. Bahn JH, Zhang Q, Li F, Chan TM, Lin X, Kim Y, Wong DT, Xiao X. The landscape of microRNA, Piwi-interacting RNA, and circular RNA in human saliva. Clin Chem. 2015;61(1):221-30.

16. Lim Y, Totsika M, Morrison M, Punyadeera C. The saliva microbiome profiles are minimally affected by collection method or DNA extraction protocols. Sci Rep. 2017;7(1):8523.

17. Marotz C, Morton JT, Navarro P, Coker J, Belda-Ferre P, Knight R, Zengler K: Quantifying live microbial load in human saliva samples over time reveals stable composition and dynamic load. mSystems 2021;6(1).

18. Sweet D, Lorente M, Valenzuela A, Lorente JA, Alvarez JC. Increasing DNA extraction yield from saliva stains with a modified Chelex method. Forensic Sci Int. 1996;83(3):167-77.

19. Bardou P, Mariette J, Escudie F, Djemiel C, Klopp C. jvenn: an interactive Venn diagram viewer. BMC Bioinformatics. 2014;15:293.

20. Yuan Y, Zheng G, Lin M, Mustapha A. Detection of viable Escherichia coli in environmental water using combined propidium monoazide staining and quantitative PCR. Water Res. 2018;145:398-407.

21. Exterkate RA, Zaura E, Buijs MJ, Koopman J, Crielaard W, ten Cate JM. The effects of propidium monoazide treatment on the measured composition of polymicrobial biofilms after treatment with chlorhexidine. Caries Res. 2014;48(4):291-8.

22. Da Collina GA, Tempestini-Horliana ACR, da Silva DFT, Longo PL, Makabe MLF, Pavani C. Oral hygiene in intensive care unit patients with 
photodynamic therapy: study protocol for randomised controlled trial. Trials. 2017;18(1):385.

23. Lagier JC, Khelaifia S, Alou MT, Ndongo S, Dione N, Hugon P, Caputo A, Cadoret F, Traore SI, Seck EH, et al. Culture of previously uncultured members of the human gut microbiota by culturomics. Nat Microbiol. 2016;1:16203.

24. Blazewicz SJ, Barnard RL, Daly RA, Firestone MK. Evaluating rRNA as an indicator of microbial activity in environmental communities: limitations and uses. ISME J. 2013;7(11):2061-8.

25. Nocker A, Sossa-Fernandez P, Burr MD, Camper AK. Use of propidium monoazide for live/dead distinction in microbial ecology. Appl Environ Microbiol. 2007;73(16):5111-7.

26. Bae $S$, Wuertz S. Discrimination of viable and dead fecal Bacteroidales bacteria by quantitative PCR with propidium monoazide. Appl Environ Microbiol. 2009;75(9):2940-4.

27. Yanez MA, Nocker A, Soria-Soria E, Murtula R, Martinez L, Catalan V. Quantification of viable Legionella pneumophila cells using propidium monoazide combined with quantitative PCR. J Microbiol Methods. 2011;85(2):124-30.

28. Munukka E, Wiklund P, Pekkala S, Volgyi E, Xu L, Cheng S, Lyytikainen A, Marjomaki V, Alen M, Vaahtovuo J, et al. Women with and without metabolic disorder differ in their gut microbiota composition. Obesity (Silver Spring). 2012;20(5):1082-7.

29. Chang WH, Wang $C H$, Lin CL, Wu JJ, Lee MS, Lee GB. Rapid detection and typing of live bacteria from human joint fluid samples by utilizing an integrated microfluidic system. Biosens Bioelectron. 2015;66:148-54.

30. Zhou H, Yang D, Ivleva NP, Mircescu NE, Schubert S, Niessner R, Wieser A, Haisch C. Label-free in situ discrimination of live and dead bacteria by surface-enhanced Raman scattering. Anal Chem. 2015;87(13):6553-61.

31. Li R, Dhankhar D, Chen J, Cesario TC, Rentzepis PM. Determination of live: dead bacteria as a function of antibiotic treatment. J Microbiol Methods. 2018;154:73-8.

32. Li R, Tun HM, Jahan M, Zhang Z, Kumar A, Dilantha Fernando WG, Farenhorst A, Khafipour E. Comparison of DNA-, PMA-, and RNA-based 165 rRNA Illumina sequencing for detection of live bacteria in water. Sci Rep. 2017;7(1):5752.

33. Stehlikova Z, Tlaskal V, Galanova N, Roubalova R, Kreisinger J, Dvorak J, Prochazkova P, Kostovcikova K, Bartova J, Libanska M, et al. Oral microbiota composition and antimicrobial antibody response in patients with recurrent aphthous stomatitis. Microorganisms. 2019;7(12):636.
34. Xu Y, Zhang M, Zhang J, Sun Z, Ran L, Ban Y, Wang B, Hou X, Zhai S, Ren L, et al. Differential intestinal and oral microbiota features associated with gestational diabetes and maternal inflammation. Am J Physiol Endocrinol Metab. 2020;319(2):E247-53.

35. Li Y, Zou CG, Fu Y, Li Y, Zhou Q, Liu B, Zhang Z, Liu J. Oral microbial community typing of caries and pigment in primary dentition. BMC Genomics. 2016;17:558

36. Kolenbrander PE, Andersen RN, Moore LV. Coaggregation of Fusobacterium nucleatum, Selenomonas flueggei, Selenomonas infelix, Selenomonas noxia, and Selenomonas sputigena with strains from 11 genera of oral bacteria. Infect Immun. 1989;57(10):3194-203.

37. Vascellari S, Palmas V, Melis M, Pisanu S, Cusano R, Uva P, Perra D, Madau V, Sarchioto M, Oppo V et al: Gut Microbiota and Metabolome Alterations Associated with Parkinson's Disease. mSystems 2020;5(5).

38. Zhao H, Chu M, Huang Z, Yang X, Ran S, Hu B, Zhang C, Liang J. Variations in oral microbiota associated with oral cancer. Sci Rep. 2017:7(1):11773.

39. Colombo AP, Boches SK, Cotton SL, Goodson JM, Kent R, Haffajee AD, Socransky SS, Hasturk H, Van Dyke TE, Dewhirst F, et al. Comparisons of subgingival microbial profiles of refractory periodontitis, severe periodontitis, and periodontal health using the human oral microbe identification microarray. J Periodontol. 2009;80(9):1421-32.

40. Yamamoto K, Honda T, Ito T, Ishizu Y, Kuzuya T, Nakamura M, Miyahara R, Kawashima H, Ishigami M, Fujishiro M. The relationship between oralorigin bacteria in the fecal microbiome and albumin-bilirubin grade in patients with hepatitis C. J Gastroenterol Hepatol. 2021;36(3):790-9.

41. Albandar JM, Khattab R, Monem F, Barbuto SM, Paster BJ. The subgingival microbiota of Papillon-Lefevre syndrome. J Periodontol. 2012;83(7):902-8.

42. Nearing JT, DeClercq V, Van Limbergen J, Langille MGI: Assessing the variation within the oral microbiome of healthy adults. mSphere 2020;5(5).

43. Kundig W, Kundig FD, Anderson B, Roseman S. Restoration of active transport of glycosides in Escherichia coli by a component of a phosphotransferase system. J Biol Chem. 1966;241(13):3243-6.

44. Netuschil L, Reich E, Unteregger G, Sculean A, Brecx M. A pilot study of confocal laser scanning microscopy for the assessment of undisturbed dental plaque vitality and topography. Arch Oral Biol. 1998;43(4):277-85.

\section{Publisher's Note}

Springer Nature remains neutral with regard to jurisdictional claims in published maps and institutional affiliations.
Ready to submit your research? Choose BMC and benefit from:

- fast, convenient online submission

- thorough peer review by experienced researchers in your field

- rapid publication on acceptance

- support for research data, including large and complex data types

- gold Open Access which fosters wider collaboration and increased citations

- maximum visibility for your research: over $100 \mathrm{M}$ website views per year

At BMC, research is always in progress.

Learn more biomedcentral.com/submissions 